The excited random walk in one dimension

This article has been downloaded from IOPscience. Please scroll down to see the full text article.

2005 J. Phys. A: Math. Gen. 382555

(http://iopscience.iop.org/0305-4470/38/12/002)

View the table of contents for this issue, or go to the journal homepage for more

Download details:

IP Address: 128.197.40.148

The article was downloaded on 06/12/2010 at 18:47

Please note that terms and conditions apply. 


\title{
The excited random walk in one dimension
}

\author{
T Antal ${ }^{1,3}$ and S Redner ${ }^{2,4}$ \\ ${ }^{1}$ Center for Polymer Studies and Department of Physics, Boston University, Boston, MA 02215, \\ USA \\ ${ }^{2}$ Theory Division and Center for Nonlinear Studies, Los Alamos National Laboratory, \\ Los Alamos, New Mexico 87545, USA \\ E-mail: redner@cnls.lanl.gov
}

Received 21 December 2004, in final form 31 January 2005

Published 9 March 2005

Online at stacks.iop.org/JPhysA/38/2555

\begin{abstract}
We study the excited random walk, in which a walk that is at a site that contains cookies eats one cookie and then hops to the right with probability $p$ and to the left with probability $q=1-p$. If the walk hops onto an empty site, there is no bias. For the 1-excited walk on the half-line (one cookie initially at each site), the probability of first returning to the starting point at time $t$ scales as $t^{-(2-p)}$. Although the average return time to the origin is infinite for all $p$, the walk eats, on average, only a finite number of cookies until this first return when $p<1 / 2$. For the infinite line, the probability distribution for the 1-excited walk has an unusual anomaly at the origin. The positions of the leftmost and rightmost uneaten cookies can be accurately estimated by probabilistic arguments and their corresponding distributions have power-law singularities. The 2-excited walk on the infinite line exhibits peculiar features in the regime $p>3 / 4$, where the walk is transient, including a mean displacement that grows as $t^{v}$, with $v>\frac{1}{2}$ dependent on $p$, and a breakdown of scaling for the probability distribution of the walk.
\end{abstract}

PACS numbers: 02.50.-r, 05.40.-a

(Some figures in this article are in colour only in the electronic version)

\section{Introduction}

In this work, we study the $k$-excited random walk in one dimension. This recently introduced model [1, 2], gives rise to a stochastic process with many unusual properties [3-6]. The definition of the excited random walk is as follows: initially each site of a one-dimensional

3 On leave from Institute for Theoretical Physics—HAS, Eötvös University, Budapest, Hungary.

4 Permanent address: Department of Physics, Boston University, Boston, MA 02215, USA. 
chain contains a fixed number of cookies $k$ (with $k \geqslant 1$ ). A random walk makes nearestneighbour hops between sites on the chain, with hopping probabilities that depend on whether or not the current site contains a cookie. When the walker hops onto a site that contains one or more cookies, the walker eats a cookie and, at the next time step, the walk hops to the right with probability $p>1 / 2$ and to the left with probability $q=1-p$. If the walker hops onto an empty site, then the hopping probabilities to the left and right at the next step are both $1 / 2$. This bias can be viewed as the foraging strategy of a primitive organism. When it encounters food, the organism consumes the food and continues to move in a preferred direction. Conversely, the organism wanders aimlessly when it does not encounter any food.

A basic feature of the excited random walk is the transitory nature of the bias; that is, when all the cookies at a given site are eaten the subsequent motion is unbiased. This non-Markovian feature is responsible for the unusual properties of the excited random walk. Related nonMarkovian constraints arise in many important stochastic phenomena. Perhaps the best known example is the self-avoiding random walk, where returns to previously visited sites are strictly forbidden [7]. This leads to an expansion, relative to the pure random walk, in which the root-mean-square displacement grows faster than $t^{1 / 2}$ when the spatial dimension is less than 4. A related example is the 'true' self-avoiding walk, where returns to previously visited sites are inhibited by an energetic constraint [8]. The opposite type of perturbation occurs in the reinforced random walk [9], where the walk has a preference to hop onto previously visited sites. Depending of the functional form of this reinforcement, the walk may, quite surprisingly, 'condense' onto a support that consists of only a finite number of sites as $t \rightarrow \infty$ [9].

The excited random walk represents a simple and surprisingly rich example of a nonMarkovian process in which the bias at each site disappears when all the cookies on the site are eaten. Because a random walk in one dimension is recurrent, that is, the probability to eventually return to the starting point equals one, the walk returns to previously visited sites infinitely often. For the 1-excited walk, the reinforcement mechanism due to the presence of cookies is insufficient to counterbalance this recurrence of the random walk; thus the 1-excited random walk remains recurrent for any $p<1$. Nevertheless, this model exhibits many novel features. For example, the probability that a 1-excited walk on an infinite line first returns to the origin at time $t$ decays as $t^{-(2-p)}$, rather than the $t^{-3 / 2}$ decay for a pure random walk $[4,10,11]$.

On the other hand, the $k$-excited random walk with $k>1$ is more strongly influenced by the cookie-induced bias. In fact, for $p>\frac{1}{2}\left(1+\frac{1}{k}\right)$, the $k$-excited random walk becomes transient [6]. This transition has dramatic consequences for the statistical properties of the walk as $p$ is varied. As a final introductory point, the excited random walk appears to be most interesting in one dimension because of the non-trivial competition between the reinforcement when a cookie is eaten and the fact that encounters with sites that contain cookies become progressively less frequent. In contrast, an unbiased random walk in greater than one dimension typically encounters previously unvisited sites [12, 13]. Because each such site contains a cookie, the reinforcement mechanism acts as a non-zero global bias that is superimposed on the random walk motion. Thus the asymptotic behaviour of an excited walk in higher dimensions is close to that of a simple biased random walk.

In the following section, we begin by recounting some basic first-passage properties for diffusion in a finite interval $[12,13]$. We present these results separately because they underlie our analytical treatment of excited random walks. In section 3, we study a particularly simple version of the excited random walk - the 1-excited walk with a reflecting boundary condition at the origin. We use basic tools of first-passage processes to determine the time dependence of the size of the region in which the cookies have been eaten and thence the probability for the walk to first return to the origin. The long-time behaviour of the first-passage 
probability in a closely related model was obtained previously, using quite different methods, in $[10,11]$. Our approach is also complementary to the tools employed in the probabilistic studies of excited random walks given in [3-6]. In section 4, we first present numerical simulations for the unusual properties for the probability distribution and for the span of the 1-excited random walk on the infinite line. We also construct a probabilistic argument to quantify the asymmetry in the number of cookies eaten from the left and right sides of the line. Finally, in section 5 we present several intriguing properties of the 2-excited walk on the infinite line, including the lack of scaling in the probability distribution, the time for the 2-excited walk to traverse a finite domain and basic features of the cookie density profile that is left behind as the walk advances.

\section{Preliminaries: first passage in an interval}

To determine the motion of excited random walks, we require the first-passage properties of a nearest-neighbour unbiased random walk, or equivalently, continuum diffusion, in a finite interval $[0, k]$ when both boundaries are absorbing. The first such property is the eventual hitting, or exit, probability of $x=0$ and of $x=k$ for a random walk that starts at $x_{0}$ within the interval $[12,13]$. These exit probabilities are

$$
E_{-}\left(k, x_{0}\right)=1-\frac{x_{0}}{k} \quad E_{+}\left(k, x_{0}\right)=\frac{x_{0}}{k} .
$$

Closely related quantities are the conditional exit times to $x=0$ and $x=k$. These are the mean times for the random walk to hit $x=0$ or to hit $x=k$ with the restriction that the other boundary is never hit. These conditional times are

$$
t_{-}\left(k, x_{0}\right)=\frac{x_{0}}{3}\left(2 k-x_{0}\right) \quad t_{+}\left(k, x_{0}\right)=\frac{1}{3}\left(k^{2}-x_{0}^{2}\right) .
$$

Next, we will also need the Laplace transforms of the first-passage probabilities for a random walk that starts at $x_{0}$ to exit at the left edge and at the right edge of the absorbing interval $[0, k]$ at time $t$. In the continuum limit, these first-passage probabilities are (equation (2.2.10) in [13])

$$
\ell_{k}\left(x_{0}, s\right)=\frac{\sinh \sqrt{2 s}\left(k-x_{0}\right)}{\sinh \sqrt{2 s} k}, \quad r_{k}\left(x_{0}, s\right)=\frac{\sinh \sqrt{2 s} x_{0}}{\sinh \sqrt{2 s} k},
$$

where we take the diffusion coefficient to be $D=1 / 2$ for the nearest-neighbour random walk.

We will also need first-passage properties in the interval $[0, k]$ when the boundary at $x=0$ is reflecting and the other boundary is absorbing. We implement the reflecting boundary condition for a discrete random walk that is at site $x=0$ by allowing the walk to remain at $x=0$ with probability $1 / 2$ or by having hop to $x=1$ with probability $1 / 2$. This definition for the reflecting boundary has the advantage that it leads to a constant probability density around this boundary for large times. With this definition, the average time to exit at the right edge of the interval $[0, k]$ as a function of $x_{0}$ is

$$
t\left(k, x_{0}\right)=k(k+1)-x_{0}\left(x_{0}+1\right) .
$$

Finally, the Laplace transform of the first-passage probability to exit at the right edge at time $t$ in the continuum limit is (equation (2.2.21) in [13])

$$
\mathcal{R}_{k}\left(x_{0}, s\right)=\frac{\cosh \sqrt{2 s}\left(k-x_{0}\right)}{\cosh \sqrt{2 s} k} .
$$

We will exploit these results to now determine various basic features of the 1-excited random walk in one dimension. There are two basic properties of the excited random walk 

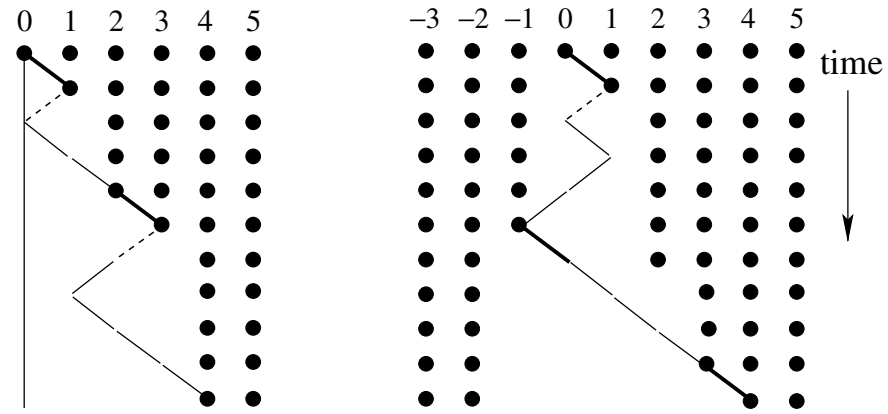

Figure 1. Space-time plot of the first few steps of a 1-excited random walk in one dimension with reflection at the origin (left), and on the infinite line (right). Cookies are shown as large dots. Steps occurring with probability $p$ and $q$ are shown as thick and dashed lines, while steps occurring with probability $1 / 2$ are shown as thin lines. For the half-line system, the last cookie at $n=4$ is first hit after 10 time steps.

that we will be able to determine analytically: (i) the location of the last (leftmost) cookie; we obtain this property in terms of the first-passage statistics of a 1-excited random walk to the right edge of the cookie-free domain, and (ii) the statistics of returns to the origin.

\section{1-excited random walk on the half-line}

Consider the 1-excited random walk that is restricted to the non-negative integers by imposing a reflecting boundary condition at the origin. Initially there is one cookie at each site and the walk is at $n=0$. At the first time step, the walk either hops to $n=1$ with probability $p$ or it remains at site $n=0$ with probability $q$ (figure 1 ). At later times, a bias in the random walk motion occurs whenever the walk reaches the right edge of the cookie-free domain. The restriction of fixing one boundary condition while the other boundary condition 'floats' greatly simplifies the analytical description of the 1-excited walk on the half-line compared to the infinite line system.

\subsection{Time to hit the last cookie}

It is useful to characterize the walk by the sequence of times $t_{n}$ at which the walk hits the leftmost (last) cookie at $n$ (figure 1). Suppose that the walk has just reached the cookie at $n-1$. Then with probability $p$, the next step of the walk is to right, which takes one time step, while with probability $q$, the walk steps left to $n-2$ and the last cookie now is at $n$. In this latter case, the average time it takes the walk to reach site $n$ is $t(n, n-2)=4 n-2$ (see equation (4)) plus the time for the initial step from $n-1$ to $n-2$. Thus the mean time increment for the last cookie to move from $n-1$ to $n$ is

$$
\left\langle\mathrm{d} t_{n}\right\rangle=p \cdot 1+q \cdot(1+4 n-2)=1+q(4 n-2) .
$$

Consequently, the mean time for the walk to hit the last cookie at $n$ for the first time is

$$
\left\langle t_{n}\right\rangle=\sum_{k=1}^{n}\left\langle\mathrm{~d} t_{k}\right\rangle=2 q n^{2}+n .
$$

Note that for $q \rightarrow 0(p \rightarrow 1)$, the early time behaviour is governed by ballistic motion, after which there is a crossover at a time of order $1 / q$ to the asymptotic diffusive behaviour of $\left\langle t_{n}\right\rangle \sim 2 q n^{2}$. 
We now extend this analysis to obtain the distribution of first-passage times for the walk to hit the last cookie at $n$ at time $t$. Let $Q_{k}(t)$ be the probability that the time interval between the walk first hitting site $k-1$ and first hitting site $k$ equals $t$. Similarly, let $F_{n}(t)$ be the first-passage probability that the walk hits site $n$ for the first time at time $t$. Then

$$
F_{n}(t)=\int \prod_{k=1}^{n} Q_{k}\left(t_{k}\right) \mathrm{d} t_{k},
$$

with $t_{1}+t_{2}+t_{3}+\cdots t_{n}=t$. Because this relation is a convolution, the Laplace transform $F_{n}(s)$ obeys

$$
F_{n}(s)=\prod_{k=1}^{n} Q_{k}(s)
$$

Now the Laplace transform of $Q_{k}(t)$ is, using equation (5),

$$
Q_{k}(s)=p(1-s)+q(1-s) \mathcal{R}_{k}(2, s) .
$$

The first term accounts for the direct step from $k-1$ to $k$. The contribution of this direct process to the discrete generating function would be $p z$. In the continuum limit, the factor $z$ maps to the Laplace variable $1-s$. The second term accounts for a single step to the left (factor $q(1-s)$ ) and the subsequent first passage from $k-2$ to $k$ in the presence of a reflecting boundary at the origin. The first step of the walk is accounted for by defining $Q_{1}(s)=e^{s}$.

We anticipate that the dominant contribution to $F_{n}(s)$ in the long-time limit comes from terms where $k \sim \sqrt{t}$ [14]. In this limit, equation (8) reduces to

$$
Q_{k}(s)=1-2 q \sqrt{2 s} \tanh \sqrt{2 s} k,
$$

by expanding $\mathcal{R}_{k}$ of equation (5) for large $k$. Using this approximation for $Q_{k}$ and taking the limit $s \rightarrow 0$, corresponding to long-time behaviour, $F_{n}(s)$ can be written as

$$
\begin{aligned}
F_{n}(s) & \approx \prod_{k=1}^{n}(1-2 q \sqrt{2 s} \tanh \sqrt{2 s} k) \\
& =\exp \left(\sum_{1}^{n} \ln (1-2 q \sqrt{2 s} \tanh \sqrt{2 s} k)\right) \\
& \sim \exp \left(-\int^{x} 2 q \sqrt{2 s} \tanh \sqrt{2 s} k \mathrm{~d} k\right) \\
& \sim(\cosh \sqrt{2 s} x)^{-2 q},
\end{aligned}
$$

where we use $\int \tanh x \mathrm{~d} x=\ln \cosh x$ and replace the discrete variable $n$ by the continuous variable $x$ in the last two lines.

From this formal solution, the cumulants $\kappa_{\ell}$ of the first-passage time may be obtained as the coefficients of $(-s)^{\ell}$ in the power series of $\ln F(x, s)$. For this we use the product representation of the cosh function, and the series expansion of $\ln (1+z)$ to give

$$
\ln F(x, s)=2 q \sum_{\ell=1}^{\infty} \frac{1}{\ell}\left(\frac{8 x^{2}}{\pi^{2}}\right)^{\ell}(-s)^{\ell} \sum_{k=0}^{\infty}(2 k+1)^{-2 \ell} .
$$

Recognizing that the second sum can be expressed in terms of the Riemann zeta function, the cumulants are

$$
\kappa_{\ell}=q x^{2 \ell} C(\ell),
$$




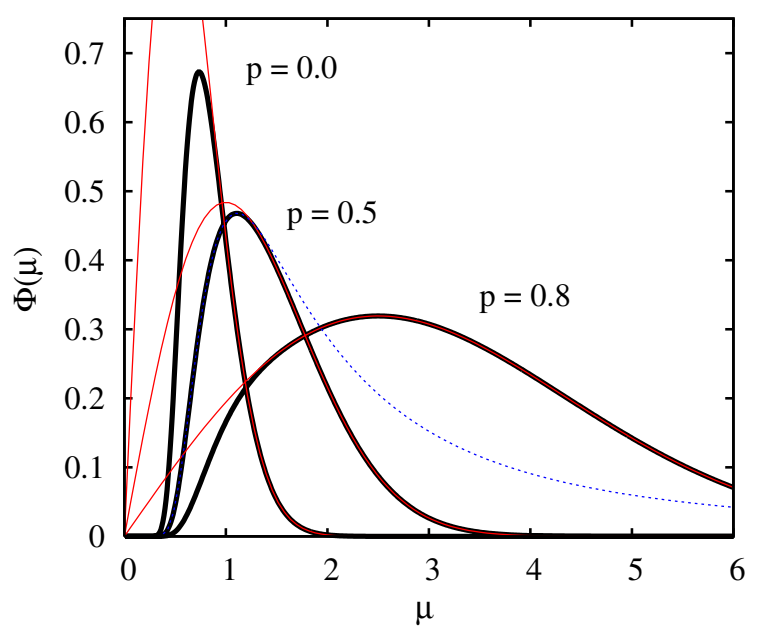

Figure 2. The scaling function $\Phi(\mu)$ in the scaling limit for $p=0,0.5$ and 0.8 . The large- $\mu$ asymptotic is represented by the first term in equation (14) (thin curves). For $p=0.5$ the first term of the small $\mu$ series of equation (12) is also shown (dotted).

where

$$
C(\ell)=8^{\ell+1 / 3}\left(1-2^{-2 \ell}\right)(\ell-1) ! \pi^{-2 \ell} \zeta(2 \ell),
$$

which gives $C(1)=2, C(2)=4 / 3, C(3)=32 / 15$, etc. This result agrees with the direct solution given in equation (6) for the average first-passage time $\langle t(x)\rangle=\kappa_{1}=2 q x^{2}$.

Finally, the time dependence of the first-passage probability for the last cookie - the probability that the cookie at $x$ is first hit at time $t$-is obtained by inverting the Laplace transform in equation (9). To simplify this calculation, we introduce the scaling variables

$$
\mu=\frac{x}{\sqrt{t}}, \quad y=s t,
$$

to rewrite the first-passage probability $F(x, t)$ in the scaling form

$$
t F(x, t) \equiv \Phi(\mu)=\frac{1}{2 \pi \mathrm{i}} \int_{-\mathrm{i} \infty}^{\mathrm{i} \infty} \mathrm{d} y \mathrm{e}^{y}(\cosh \sqrt{2 y} \mu)^{-2 q} .
$$

For the case of no bias ( $p=q=1 / 2$ ), the integrand has simple poles on the negative real axis at

$$
y=-\frac{8 y \mu^{2}}{(2 k+1)^{2} \pi^{2}}, \quad k=0,1, \ldots
$$

By evaluating the corresponding residues at these poles, we find the scaling function

$$
\Phi_{p=\frac{1}{2}}(\mu)=\frac{\pi}{2 \mu^{2}} \sum_{k=0}^{\infty}(2 k+1)(-1)^{k} \mathrm{e}^{-(2 k+1)^{2} \pi^{2} / 8 \mu^{2}} .
$$

Because of the presence of an exponential cutoff, this series is easy to compute numerically and the result is displayed in figure 2, together with the small- $\mu$ limiting behaviour (corresponding to small $x$ for fixed $t$ or large $t$ for fixed $x$ ) that stems from the first term of equation (12):

$$
\Phi_{p=\frac{1}{2}}(\mu) \approx \frac{\pi}{2 \mu^{2}} \mathrm{e}^{-\frac{\pi^{2}}{8 \mu^{2}}} .
$$




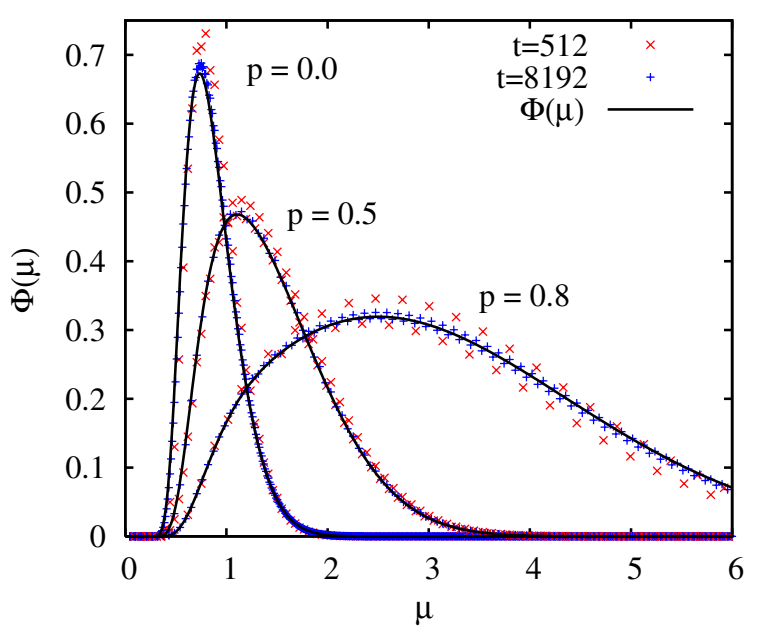

Figure 3. The scaling function compared to the numerical exact propagation of the probability distribution.

For general $p \neq 1 / 2$ the poles in equation (11) are branch points and we therefore evaluate this integral in a simpler manner by first using the binomial theorem to write equation (9) as

$$
F(x, s)=2^{2 q} \sum_{k=0}^{\infty}\left(\begin{array}{c}
-2 q \\
k
\end{array}\right) \mathrm{e}^{-2 x \sqrt{2 s}(k+q)} .
$$

Each term in this series can be easily back transformed term by term (see 29.3.82 in [16]). The scaling function thus has the form

$$
\Phi(\mu)=\mu \frac{2^{2 q+1 / 2}}{\sqrt{\pi}} \sum_{k=0}^{\infty}\left(\begin{array}{c}
-2 q \\
k
\end{array}\right)(k+q) \mathrm{e}^{-2 \mu^{2}(k+q)^{2}} .
$$

For $q=1 / 2$, this function is identical to $\Phi(\mu)$ in equation (12) in spite of the apparent visual differences in these two formulae, since both functions originate from the same Laplace transform. Equation (14) can be viewed as the large- $\mu$ expansion of $\Phi(\mu)$. As shown in figure 2 , the $k=0$ term in this series representation

$$
\Phi(\mu) \approx \mu q \frac{2^{2 q+1 / 2}}{\sqrt{\pi}} \mathrm{e}^{-2(\mu q)^{2}},
$$

together with the $k=0$ term in the small- $\mu$ series for equation (12) give a fairly good approximation for $\Phi(\mu)$ over the entire range of $\mu$ for $p=1 / 2$. Figure 3 shows the solution to equation (14), together with the exact numerical propagation of the probability distribution at different times (see appendix).

\subsection{Location of the last cookie}

Another important characterization of the excited random walk is the probability distribution for the location of the last cookie. Now the last cookie either remains stationary or it moves only to the right during each step of the underlying excited random walk. Consequently, we can write a simple relation between the first-passage probability for hitting the last cookie, derived in the previous section and the probability distribution of this last cookie. 
The probability that the last cookie is located at site $n$ at time $t$ equals the joint probability that a walk first hopped to site $n-1$ before time $t$, and that this walk then first hopped to site $n$ after time $t$. That is,

$$
P_{n}(t)=\sum_{t^{\prime}=1}^{t} \sum_{t^{\prime \prime}=t-t^{\prime}+1}^{\infty} F_{n-1}\left(t^{\prime}\right) Q_{n}\left(t^{\prime \prime}\right) .
$$

In the continuum limit, the Laplace transform of this equation is

$$
P_{n}(s)=\frac{1}{s} F_{n-1}(s)\left[1-Q_{n}(s)\right]=\frac{1}{s}\left[F_{n-1}(s)-F_{n}(s)\right],
$$

where we used equation (7) to simplify the last factor. In the continuous space limit, the relation between $P$ and $F$ becomes

$$
P(x, s)=-\frac{1}{s} \frac{\partial F(x, s)}{\partial x},
$$

which is equivalent to

$$
P(x, t)=-\frac{\partial}{\partial x} \int_{0}^{t} \mathrm{~d} t^{\prime} F\left(x, t^{\prime}\right) .
$$

Substituting the scaling form for $F(x, t)$ into equation (17) and performing the derivative, we express the integrand in scaled units, after which the integral can be evaluated easily.

These steps lead to the following simple connection between the first-passage and occupation probabilities of the last cookie:

$$
F(x, t)=\frac{1}{t} \Phi\left(\frac{x}{\sqrt{t}}\right), \quad \text { and } \quad P(x, t)=\frac{2}{x} \Phi\left(\frac{x}{\sqrt{t}}\right),
$$

with the same scaling function for both quantities. We exploit this connection between $F$ and $P$ to obtain the finite time data in figure 3 by first calculating the position distribution of the last cookie from the numerical evaluation of the Master equation (see appendix), and then using equation (18) to arrive at the scaling function $\Phi(\mu)$.

Finally, we use equations (14) and (18) to obtain the exact expression for the average position of the last cookie

$$
\langle x(t)\rangle=\int_{0}^{\infty} \mathrm{d} x \times P(x, t) \equiv 2 \sqrt{t} \mathcal{N}(q),
$$

where the proportionality constant

$$
\mathcal{N}(q)=\int_{0}^{\infty} \mathrm{d} \mu \Phi(\mu)=\frac{2^{2 q-3 / 2}}{\sqrt{\pi} q} \frac{[\Gamma(1+q)]^{2}}{\Gamma(1+2 q)}
$$

is obtained by first integrating (14) term by term and then using the identity [17]

$$
\sum_{k=0}^{\infty}\left(\begin{array}{c}
-2 q \\
k
\end{array}\right) \frac{1}{k+q}=\frac{[\Gamma(1+q)]^{2}}{q \Gamma(1+2 q)} .
$$

The surprising point of this calculation is that $\langle x(t)\rangle \sim \sqrt{t} / q$ as $q \rightarrow 0$, while the mean first-passage time for the walk to reach a distance $x$ scales as $\langle t(x)\rangle \propto q x^{2}$. More precisely,

$$
\langle t(x)\rangle=2 q x^{2}, \quad\langle x(t)\rangle \sim \frac{1}{\sqrt{2 \pi} q} \sqrt{t},
$$

while naive dimensional scaling would suggest that $\langle t\rangle \propto q x^{2}$ implies $\langle x(t)\rangle \propto \sqrt{t / q}$. The fact that this naive scaling does not hold is a result of the broadness of the distribution of first-passage times for the last cookie. 


\subsection{First passage to the origin}

As a complement to the statistical properties of the last cookie, we now study first passage to the origin, namely, the probability for the 1-excited walk to reach $x=0$ for the first time at time $t$. We first provide a heuristic argument (previously given in [4]) that the 1-excited walk is recurrent and then we extend this line of reasoning to argue that the first-passage probability asymptotically scales at $t^{-q-1}$.

If the walk is at a point $x-1>0$ for the first time, then the probability for the walk to subsequently reach $x$ without hitting the origin is $p+q\left(1-\frac{2}{x}\right)=1-\frac{2 q}{x}$. Here the factor $p$ accounts for the event where the walk immediately eats a cookie by making a step to the right, and the factor $q\left(1-\frac{2}{x}\right)$ (see equation (1)) accounts for the probability that the first step is to the left and that the walk eventually eats another cookie on the right without hitting the origin. Thus, if the walk starts at site $x=1$ with the system initially full of cookies, the probability for the walk to reach (at least) an arbitrary point $x>1$ without hitting the origin is

$$
\begin{aligned}
P(x) & =\prod_{k=2}^{x}\left(1-\frac{2 q}{k}\right)=\exp \left[\sum_{k=2}^{x} \ln \left(1-\frac{2 q}{k}\right)\right] \\
& \sim \exp \left(-\int^{x} \frac{2 q}{y} \mathrm{~d} y\right) \\
& \sim x^{-2 q} .
\end{aligned}
$$

Since $x^{-2 q} \rightarrow 0$ as $x \rightarrow \infty$, the random walk must necessarily return to the origin; that is, the 1-excited random walk is recurrent, unless $p=1$.

Since $P(x)$ is the probability that the walk eats at least $x$ cookies without hitting the origin, $P^{\prime}(x) \sim x^{-1-2 q}$ is the probability that the walk eats exactly $k$ cookies. The average number of cookies eaten by a walk that does not hit the origin is therefore $\langle n\rangle \sim \int^{\infty} \mathrm{d} x x^{-2 q}$. Thus for $q>1 / 2(p<1 / 2)$ a walk that manages to avoid the origin typically wanders only a finite distance away (for a more rigorous argument see section 4.3).

If the walk is at site $x-1$ for the first time, the conditional average time to reach site $x$ without hitting the origin is one with probability $p$, and $\frac{4}{3}(x-1)$ with probability $q$ (see equation (2)), that is $\left\langle\mathrm{d} t_{x}\right\rangle=1+\frac{4 q}{3}(x-1)$. Thus the average time to reach site $x$, without hitting the origin, is $\left\langle t_{x}\right\rangle=1+\sum_{k=3}^{x}\left\langle\mathrm{~d} t_{k}\right\rangle \sim x^{2}$ for large $x$. From this relation between $t$ and $x$, we infer that the survival probability, namely, the probability that the walk does not reach the origin by time $t$, should vary as $S(t)=P(x) \sim x^{-2 q} \sim t^{-q}$. Consequently, the first-passage probability $F(t)=-\frac{\partial S}{\partial t}$ should asymptotically decay as

$$
F(t) \sim t^{-q-1}
$$

For $q<1$, the average time to reach the origin is infinite. Quite strangely, although it takes infinite time for the walk to hit the origin for any $q$, the walk eats only a finite number of cookies, i.e., wanders only a finite distance from the origin, for $q>1 / 2$. This result remains valid even if there is an initial cookie-free region at the origin, as this initial condition would affect only the pre-asymptotic factors in equation (21).

To compute the first-passage probability to the origin more precisely, it is useful to define two auxiliary quantities, $L_{k}(t)$ and $R_{k}(t)$, as follows: assuming the walk hits site $k$ for the first time at time $t_{0}$, let

- $L_{k}(t) \equiv$ the probability of hitting site 0 for the first time at time $t_{0}+t$, without hitting $k+1$,

- $R_{k}(t) \equiv$ the probability of hitting $k=k+1$ for the first time at time $t_{0}+t$, without hitting site 0 . 
In the Laplace domain, these first-passage probabilities are, using equation (3),

$$
\begin{aligned}
& L_{k}=q(1-s) \ell_{k+1}(k-1, s), \\
& R_{k}=p(1-s)+q(1-s) r_{k+1}(k-1, s) .
\end{aligned}
$$

For example, for $L_{k}$, the first step must be to the left (factor $q(1-s)$ ) and then the factor $\ell_{k+1}$ is the first-passage probability to the origin, without hitting $k+1$, for a walk that starts at $k-1$. Similarly, for $R_{k}$, the term $p(1-s)$ accounts for the transition $k \rightarrow k+1$ in a single step. The factor $q(1-s)$ in the second term accounts for a single step to the left after which the walk is at $k-1$ while the last cookie is at $k+1$. Then the factor $r_{k+1}(k-1, s)$ gives the first-passage probability to $k+1$ without hitting the origin, when starting from $k-1$.

Finally, we obtain the Laplace transform of the first-passage probability to the origin for a walk that begins at $x=1$ with the system initially full of cookies by summing over all paths that contain $0,1,2, \ldots$ first passages to the last cookie before the origin is reached. This gives

$$
\begin{aligned}
F(s) & =L_{1}+R_{1}\left(L_{2}+R_{2}\left(L_{3}+R_{3}\left(L_{4}+\cdots\right.\right.\right. \\
& =L_{1}+R_{1} L_{2}+R_{1} R_{2} L_{3}+R_{1} R_{2} R_{3} L_{4}+\cdots .
\end{aligned}
$$

In the limit $s=0$, this Laplace transform is just the integral of the first-passage probability over all time or, equivalently, the probability of eventual return to the origin. It is easy to verify that this return probability equals one. In the $s \rightarrow 0$ limit, the auxiliary probabilities $R_{k}$ and $L_{k}$ are

$$
R_{k}=p+q \frac{k-1}{k+1}=1-\frac{2 q}{k+1} ; \quad L_{k}=\frac{2 q}{k+1}=1-R_{k} .
$$

Then

$$
F(s=0)=1-R_{1}+R_{1}\left(1-R_{2}\right)+R_{1} R_{2}\left(1-R_{3}\right)+R_{1} R_{2} R_{3}\left(1-R_{4}\right)+\cdots .
$$

This expression obviously equals one for all $p<1$, while for extreme case of $p=1, F(s=$ $0)=0$. Thus eventual return to the origin is certain for any bias $p<1$.

More formally, we denote the sum of the first $m$ terms of equation (23) as

$$
F_{m}(s)=\sum_{k=1}^{m} L_{k} \prod_{j=1}^{k-1} R_{j} .
$$

For finite $m$, we use equation (24) to give, in the limit $s \rightarrow 0$,

$$
F_{m}(s=0)=\frac{2 q}{\Gamma(2-2 q)} \sum_{k=1}^{m} \frac{\Gamma(k+1-2 q)}{\Gamma(k+2)},
$$

which goes to one as $m \rightarrow \infty$ [15]. The use of the $s=0$ forms for $R_{k}$ and $L_{k}$ from (24) remains valid for $m$ of the order of $1 / \sqrt{s}$ or less. We now assume that the main contribution to $F(s)$ comes from terms with $m<1 / \sqrt{s}$ in equation (26). That is,

$$
F(s) \approx \frac{2 q}{\Gamma(2-2 q)} \sum_{k=1}^{1 / \sqrt{s}} \frac{\Gamma(k+1-2 q)}{\Gamma(k+2)} .
$$

This finite sum can be written as the difference of the infinite sum (which equals 1 ) and the sum from $1 / \sqrt{s}$ to $\infty$. In this second sum, $k \gg 1$ for all terms as $s \rightarrow \infty$. Therefore we can replace the ratio of gamma functions by $k^{a-b}$ and the sum by an integral. These steps lead to

$$
1-F(s) \sim \int_{1 / \sqrt{s}}^{\infty} \mathrm{d} k k^{-2 q-1} \sim s^{q} .
$$

This result for $F(s)$ implies that for large times the first-passage probability to the origin indeed behaves as $F(t) \sim t^{-1-q}$. 


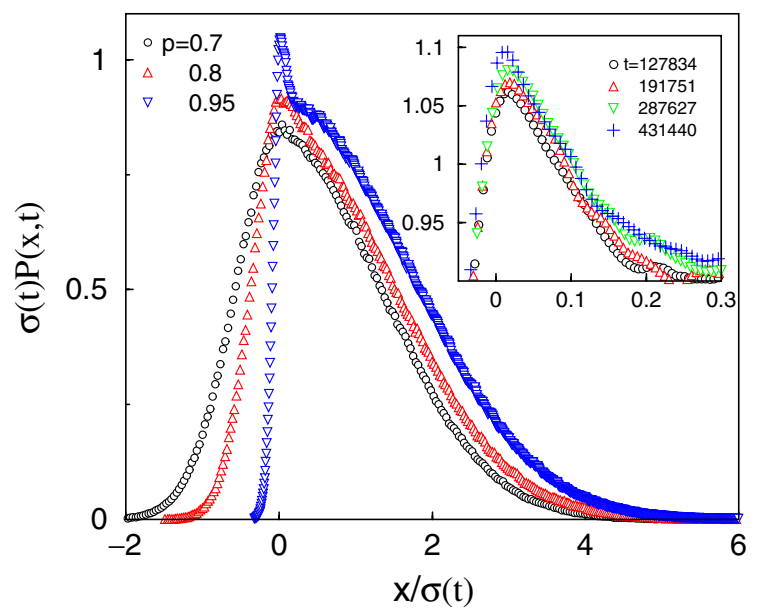

Figure 4. Scaled probability distributions of the 1-excited random walk for $2 \times 10^{6}$ realizations for $85223 \approx 1.5^{28}$ time steps for $p=0.7,0.8$ and 0.95 . Plotted on the horizontal axis is $x / \sigma$ and on the vertical axis is $\sigma P(x, t)$, where $P(x, t)$ is the probability distribution of the walk. The data have been averaged over a $\frac{1}{2} \%$ range. The inset shows the distribution for $p=0.95$ near the origin for still longer times: $t=1.5^{k}$, with $k=29-32$ (127834-431440 steps).

\section{1-excited walk on the infinite line}

\subsection{First-passage probabilities}

Using the same first-passage analysis as that given for the 1-excited walk on the half-line, we can immediately deduce the first-passage probabilities for the 1-excited walk on the infinite line to hit the left side and the right side of a cookie-free region. Thus the first-passage probability for the walk to hit the left side of this gap is the same as the first-passage probability to the origin for the half-line system. From equation (22), $F_{L}(t) \sim t^{-1-q}$, where we now write the subscript $L$ to denote hitting the left side of the gap. The first-passage probability to the right side of the gap can immediately be obtained merely by interchanging $p$ and $q$; thus $F_{R}(t) \sim t^{-1-p}$. Since the exponents in both of these first-passage probabilities are smaller than 2 , the mean time to hit either edge of the cookie-free domain is infinite.

\subsection{The probability distribution}

Another basic characteristic of the 1-excited walk is the probability distribution of displacements. An analytical treatment of this quantity is more involved than the semiinfinite system because of the existence of two moving boundaries. We thus turn to numerical simulations to provide a qualitative picture of this distribution.

Figure 4 shows the scaled probability distribution for $p=0.7,0.8$ and 0.95 . For $p \gtrsim 0.5$, the probability distribution has a nearly Gaussian, but slightly asymmetric, appearance. As $p$ increases toward 1 , this asymmetry becomes more pronounced, as shown in the figure. In addition, an apparent singularity in the distribution develops near the origin as $p \rightarrow 1$. By running simulations for $p=0.7$ and 0.95 to $431,440 \approx 1.5^{32}$ steps, we observe quite accurate data collapse for $p=0.7$. However for $p=0.95$, there is a small but persistent departure from data collapse for $0 \lesssim x / \sigma \lesssim 0.3$ (inset to figure 4). We do not understand whether 
this anomaly arises from a slow correction to scaling term in the probability distribution or whether there exists an effect that lies outside of scaling.

For all values of $p>1 / 2$, both $\langle x(t)\rangle$ and the variance $\sigma(t)=\sqrt{\left\langle x(t)^{2}\right\rangle-\langle x(t)\rangle^{2}}$ grow as $t^{1 / 2}$. The time dependence for the displacement is easy to understand on heuristic grounds. Once a cookie-free domain of linear dimension $L$ has been created, the domain then grows only when the walk reaches either edge of the domain. Since the motion of the walk is purely random within the domain, the probability that the walk hits either edge is proportional to $1 / L$. If the walk hits the right edge of the domain, then the walk will move ballistically for typically $1 /(1-p)=1 / q$ steps as $p \rightarrow 1$. Thus the equation of motion for the domain length will be $\dot{L}=1 /(L q)$ as $p \rightarrow 1$. The solution to this equation gives $L \propto t^{1 / 2}$ for any value of $p<1$. However, it bears mention that this naive approach gives the wrong value for the amplitude $-1 / \sqrt{q}-$ rather than $1 / q$ that was derived in equation (20).

\subsection{Asymmetry}

A useful geometric characteristic of the 1-excited walk is the asymmetry in the span of the walk. Here the span is the length of the cookie-free region. For an unbiased random walk, the growth of the span with time and its distribution are well understood [18], and we now investigate how the bias inherent in the 1-excited walk influences the span. It is useful to characterize the span by the positions of the last cookie on the right and the last cookie on the left of the cookie-free region. We can understand the motion of these extreme cookies by the following heuristic argument. Consider the situation where the cookie-free gap has length $L-2$ and where the walk has just stepped to the extreme cookie on the right. As shown at the beginning of section 3.3 , the probability that the walk eats another right cookie without eating a left cookie is $1-\frac{2 q}{L}$.

With this basic result, the probability that the walk eats precisely $r>0$ consecutive cookies (we term this event a single 'meal') from the right edge of the cookie-free region is

$$
\begin{aligned}
P(r) & =\prod_{j=0}^{r-2}\left(1-\frac{2 q}{L+j}\right) \times \frac{2 q}{L+r-1} \\
& =2 q \frac{\Gamma(L)}{\Gamma(L-2 q)} \frac{\Gamma(L+r-1-2 q)}{\Gamma(L+r)},
\end{aligned}
$$

where we use the convention $\prod_{a}^{b}=1$ for $a>b$. The probability that the walk eats precisely one cookie (that at the starting position) is then $2 q / L$.

When the initial cookie-free gap is large, this probability simplifies to

$$
P(r) \sim 2 q L^{2 q}(L+r)^{-2 q-1} .
$$

The $L$ dependence can be scaled out using the dimensionless variable $\tilde{r}=r / L$ and the rescaled probability

$$
\tilde{P}(\tilde{r}) \equiv L P(r)=2 q(1+\tilde{r})^{-2 q-1} .
$$

Note that this probability is normalized, $\int_{0}^{\infty} \mathrm{d} \tilde{r} \tilde{P}(\tilde{r})=1$. Using this probability, the average relative number of consecutive cookies eaten from the right side of the gap, $\int_{0}^{\infty} \mathrm{d} \tilde{r} \tilde{r} \tilde{P}(\tilde{r})$, is

$$
\langle\tilde{r}\rangle=\langle r / L\rangle= \begin{cases}\frac{1}{2 q-1}, & q>1 / 2 \\ \infty, & q \leqslant 1 / 2\end{cases}
$$

This result is paradoxical at first sight because, as we showed in section 3.3 , the average time to return to the left side of the gap is infinite. However, the walk eats, on average, only a finite 
number of cookies if $q \leqslant 1 / 2$, even though the time taken for its meal on the right side of the gap is infinite.

The average relative number of eaten cookies from the left $\langle\tilde{l}\rangle$ is also given by equation (32) after interchanging $q$ and $p$. Although the 1-excited walk eats, on average, infinitely many consecutive cookies from a single side of the gap during a single meal, the average ratio $\langle r /(l+r)\rangle$ turns out to be finite for any value of $q$. To show this we compute the joint probability of eating $r$ consecutive cookies on the right and then $l$ consecutive cookies on the left before the next right cookie is eaten, under the initial condition that the walk starts at the position of the right extreme cookie. This joint probability can be derived analogously to equation (30)

$$
\begin{aligned}
P(r, l)=4 p q & \frac{\Gamma(L)}{\Gamma(L-2 p)} \times \frac{\Gamma(L+l-1-2 p)}{\Gamma(L+l-2 q)} \frac{\Gamma(L+l+r-1-2 q)}{\Gamma(L+l+r)} \\
& \sim 4 q(1-q) L^{2 q}(L+r)^{1-4 q}(L+r+l)^{2 q-3} .
\end{aligned}
$$

It is again useful to define the rescaled variables $\tilde{l}=l / L$ and $\tilde{r}=r / L$, as well as the rescaled probability

$$
\tilde{P}(\tilde{r}, \tilde{s}) \equiv L^{2} P(r, l)=4 q(1-q)(1+\tilde{r})^{1-4 q}(1+\tilde{s})^{2 q-3},
$$

where $\tilde{s}=\tilde{l}+\tilde{r}$ is the total number of cookies eaten in one right-left cycle. One may check that this probability is normalized, $\int_{0}^{\infty} \mathrm{d} s \int_{0}^{s} \mathrm{~d} r \tilde{P}(r, s)=1$.

Finally, the average ratio for the number of right cookies to total cookies eaten in a single cycle is

$$
\begin{aligned}
\left\langle\begin{array}{l}
r \\
s
\end{array}\right\rangle & =\left\langle\begin{array}{l}
\tilde{r} \\
\tilde{\tilde{s}}
\end{array}\right\rangle=\int_{0}^{\infty} \mathrm{d} s \int_{0}^{s} \mathrm{~d} r \frac{r}{s} \tilde{P}(r, s) \\
& =4 q(1-q) \int_{0}^{\infty} \frac{\mathrm{d} s}{s}(1+s)^{2 q-3} \int_{0}^{s} \mathrm{~d} r r(1+r)^{1-4 q} .
\end{aligned}
$$

This integral can be performed with the help of 3.235 in [19] (both for $q<3 / 4$ and $q>3 / 4$ ), and also using some basic properties of the Euler $\psi$ function [16] to give

$$
\left\langle\begin{array}{l}
r \\
s
\end{array}\right\rangle=1-\frac{2 q^{2}}{(1-2 q)^{2}}-\frac{2 \pi q(1-q)}{(1-2 q)(3-4 q)} \cot 2 \pi q .
$$

This gives the asymptotically exact ratio for the number of right cookies to total cookies eaten in one cycle, under the assumption that the initial cookie-free gap is large. Although $\langle l\rangle$ or $\langle r\rangle$ is infinite, the $q$-dependence of the ratio $\langle r /(l+r)\rangle$ is well behaved (figure 5). An interesting feature of the monotonlically decreasing function $\langle r / s\rangle$ is that it goes to $2 / 3$ as $q \rightarrow 0$.

Despite the fact that the average number of consecutive cookies eaten from the left or the right side of the gap in one cycle is infinite, our data clearly show that the positions of the two extreme cookies both grow as $t^{1 / 2}$, but with very different amplitudes. To obtain an approximation for these amplitudes we now calculate the typical number $l^{*}$ and $r^{*}$ of eaten consecutive cookies from each side.

We estimate the value of $r^{*}$ by setting the probability of eating at least $r^{*}$ consecutive cookies on the right equal to the probability of going to the left before $r^{*}$ right cookies are eaten. That is,

$$
\prod_{j=0}^{r^{*}-2}\left(1-\frac{2 q}{L+j}\right)=\frac{1}{2}
$$

This criterion gives a crude estimate for the number of right cookies eaten during a single meal. For $L \gg 1$, equation (37) becomes

$$
\left(\frac{L}{L+r^{*}}\right)^{2 q}=\frac{1}{2}
$$




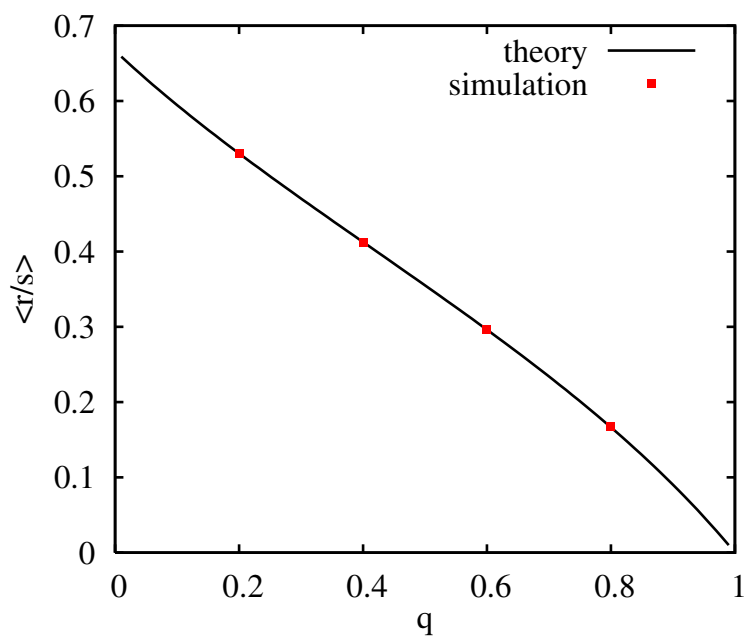

Figure 5. Ratio of the number of right cookies eaten to total cookies eaten after a single cycle (36). The squares are obtained from simulations with an initial gap ranging from 20 to 200 .

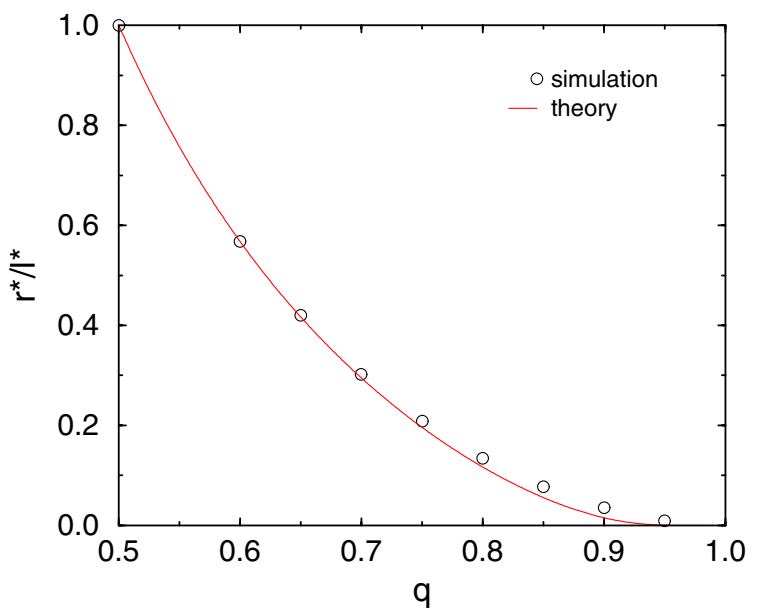

Figure 6. The ratio $r^{*} / l^{*}$ as a function of $p$ for the 1-excited random walk. The smooth curve is the prediction from equation (38), while the data are based on $2 \times 10^{6}$ realizations of the walk.

which leads to the estimate that the typical length of the string of eaten right cookies is $r^{*}=\left(2^{1 / 2 q}-1\right) L$. Applying this same argument to the left side of the cookie-free region, a string of $l^{*}=\left(2^{1 / 2 p}-1\right) L$ consecutive left cookies are typically eaten. Their ratio thus equals

$$
\frac{r^{*}}{l^{*}}=\frac{2^{1 / 2 q}-1}{2^{1 / 2 p}-1} \text {. }
$$

As a function of time, $r^{*} / l^{*}$ clearly approaches a constant value as $t \rightarrow \infty$ and it is easy to estimate its asymptotic value which is shown in figure 6 , together with the prediction of equation (38).

To conclude this section, we point out another intriguing feature of the extreme cookies; namely the probability distribution in their positions. Quite strikingly, these distributions have short-distance power-law tails, both for the left and for the right extreme cookies (figure 7) for 


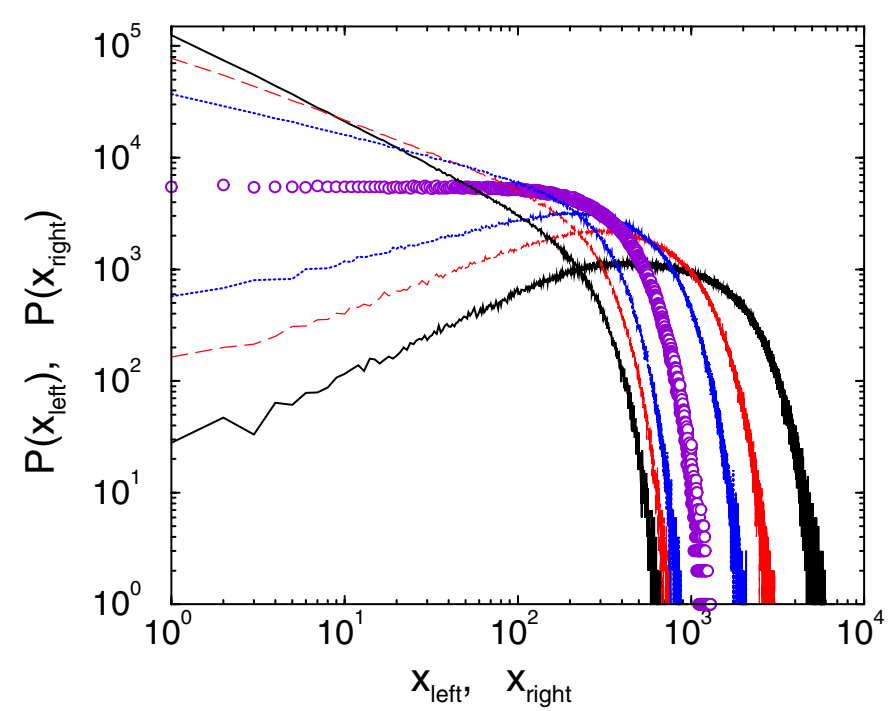

Figure 7. Probability distributions for the position of the rightmost cookie (positive slopes for small $x$ ) and the leftmost cookie for the 1-excited random walk. Data are based on $2 \times 10^{6}$ realizations for 85223 time steps, for $p=0.7$ (line), 0.8 (dashed) and 0.9 (dotted). For the rightmost cookie, the apparent power law for small $x$ has exponent $0.35,0.54,0.74$ and 0.84 for $p=0.7,0.8,0.9$ and 0.95 , respectively, while for the leftmost cookie, the respective exponents are $-0.42,-0.63,-0.83$ and -0.94 . Also shown are the data for the unbiased random walk (o).

$p \neq \frac{1}{2}$. In contrast, for the pure random walk case of $p=\frac{1}{2}$, the extreme cookie distribution is essentially the same as the span distribution, which is known to be a Gaussian-like function [18]. The origin of the short-distance power-law tails in the probability distributions of the last cookie seem to be connected with the anomalous scaling of the first-passage probability, but we do not have a definitive explanation for this phenomenon.

\section{2-excited random walk}

In the 2-excited random walk, each lattice site initially has two cookies and the $(p, q)$ bias occurs as long as the walk lands on a site that contains at least one cookie. This 2-excited random walk undergoes a transition between recurrence and transience as $p$ increases beyond a critical value $p_{c}=\frac{3}{4}$ [6]. Indeed, the $k$-excited walk for any $k>1$ appears to have qualitatively similar behaviour as the 2-excited walk. For simplicity, we thus focus on the 2-excited random walk.

We first recount the qualitative argument for the transition between recurrence and transience of the $k$-excited walk that was given in [6]. Whenever the walk is in a region that contains cookies, there is ostensibly a bias velocity $v=p-q$. Thus the time that the walk takes to travel a distance $L$ should be $L /(p-q)$. If this time is less than the total number of cookies $k L$ in this region, then the walk does not have sufficient time to consume all the cookies as it moves. Consequently, the bias will persist forever and the walk will be transient. The condition for transience is therefore $L / v<k L$, or

$$
p>p_{c}=\frac{1}{2}\left(1+\frac{1}{k}\right) .
$$




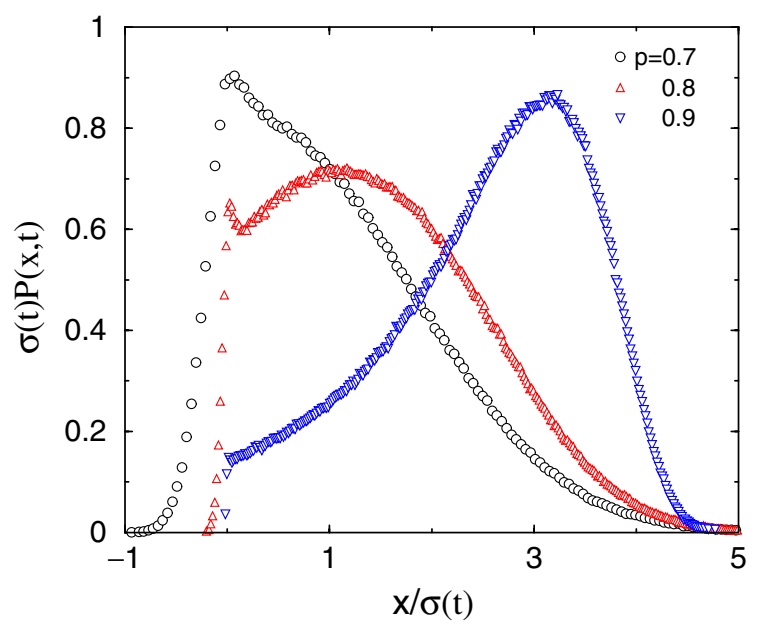

Figure 8. Scaled probability distributions of the 2-excited random walk for 85223 time steps and for $2 \times 10^{6}$ realizations. The data have been averaged over a $\frac{1}{2} \%$ range.

As we shall see, the behaviour of the 2-excited walk is qualitatively different in the regimes $p<p_{c}$ and $p>p_{c}$.

\subsection{Probability distributions}

Figure 8 shows scaled displacement probability distributions for the 2-excited random walk for representative values of $p$. As $p$ passes through $p_{c}$, there is a sudden drop in the integrated probability that the walk lies in the range $x<0$. Another basic feature of the distribution is the departure from scaling, as shown in figure 9. For $p=0.9$, data collapse has not yet set in by $431400 \approx 1.5^{32}$ time steps. If scaling were to hold, it would only arise after an extraordinarily long time. In the absence of such a clearly identifiable long-time scale, it seems that the asymptotic distribution will not obey single-parameter scaling.

The transition in the behaviour of the probability distribution is mirrored in the spatial profile of the uneaten cookies. For $p<p_{c}$, the 2 -excited random walk is recurrent and thus the region close to the origin should ultimately be devoid of cookies. This behaviour is illustrated in figure 10, where the scaled density of uneaten cookies is shown. For $p=0.6$ this density profile follows single-parameter scaling and also decays to zero near the origin because of the recurrence of the walk for $p<3 / 4$. On the other hand, for $p=0.9$, the cookie density shows a very small departure from scaling and the density goes to a non-zero limit near the origin as a result of the transient nature of the walk. We can understand this limiting cookie density near the origin by the same argument that led to the criterion for $p_{c}$ in equation (39). For general $p>p_{c}$, a walk typically resides for a time $1 /(p-q)$ at each site and should eat $1 /(p-q)$ cookies, thus leaving a residue of $2-1 /(p-q)$ uneaten cookies per site. For the case $p=0.9$ shown in figure 10 , we therefore expect $3 / 4$ of a cookie per site near the origin.

A more detailed characterization of the cookie profile is provided by the size distributions of domains that contain one cookie and contain zero cookies. The distribution of one-cookie domains is an exponentially decaying function of domain length (figure 11). This behaviour is simple to understand. The process that gives rise to a one-cookie domain as $p \rightarrow 1$ is a walk that makes a single step to the left after a run of consecutive steps to the right. During 


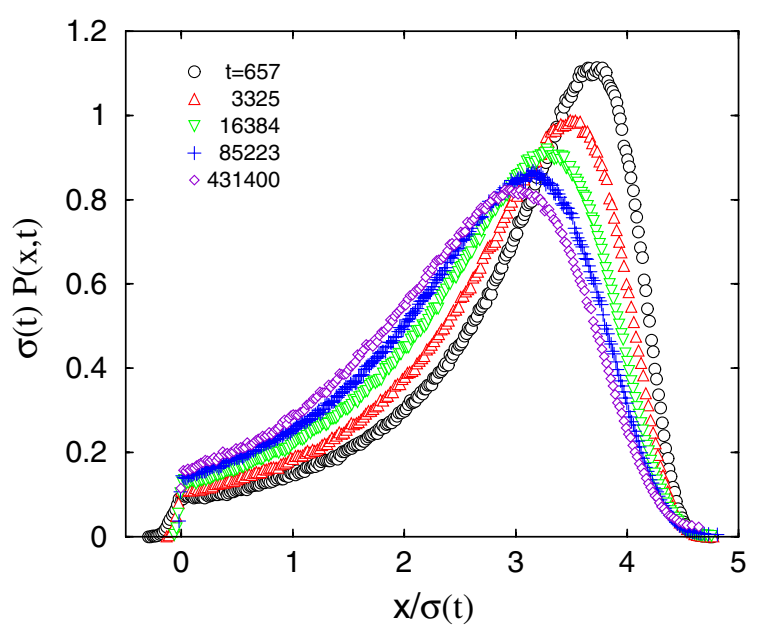

Figure 9. Scaled probability distributions of the 2-excited random walk for $p=0.9$ at different times for $2 \times 10^{6}$ realizations. The data have been averaged over a $\frac{1}{2} \%$ range.

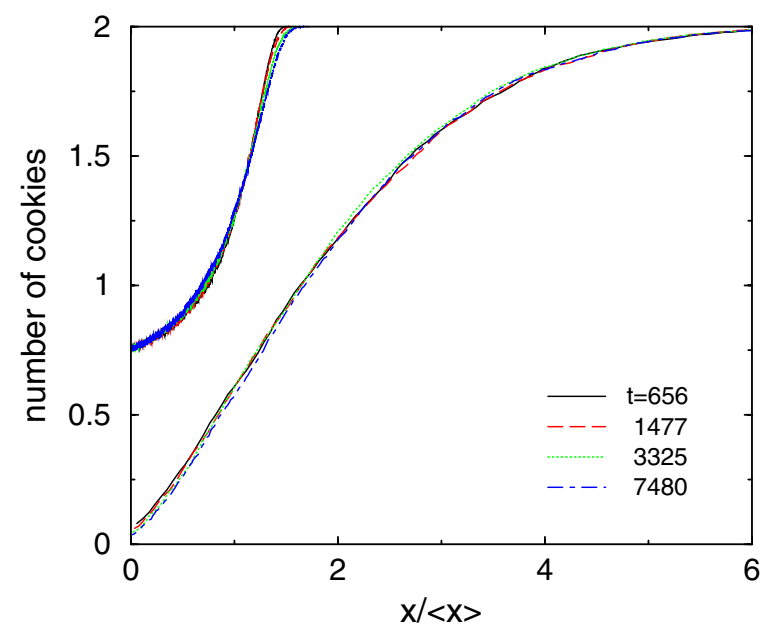

Figure 10. Cookie profile for the 2-excited random walk at $p=0.6$ (lower data) and at $p=0.9$ (upper data) for times 656,1477, 3325 and 7480. The $x$-axis has been scaled by the average displacement of the walk at time $t$.

this run of rightward steps into previously unvisited territory (each occurring with probability $p$ ), one cookie is left behind at each site. A step to the left (which occurs with probability $q$ ) terminates this one-cookie domain by a cookie-free gap. Thus the probability of a one-cookie domain of length $k$ is simply $p^{k} q=q \mathrm{e}^{-k \ln (1 / p)}$. From linear least-squares fits to the data in figure 11 , the slopes for $p=0.6,0.7,0.8$ and 0.9 are, respectively, $-0.472,-0.348,-0.219$ and -0.104 . On the other hand, the corresponding slopes from the distribution $q \mathrm{e}^{-k \ln (1 / p)}$ are $-0.511,-0.357,-0.223$ and -0.105 , are in excellent agreement with the data, especially as $p \rightarrow 1$.

The length distribution of zero-cookie domains is more intriguing (figure 12). Such domains arise both by the growth of isolated zero-cookie domain and by the merging of two 


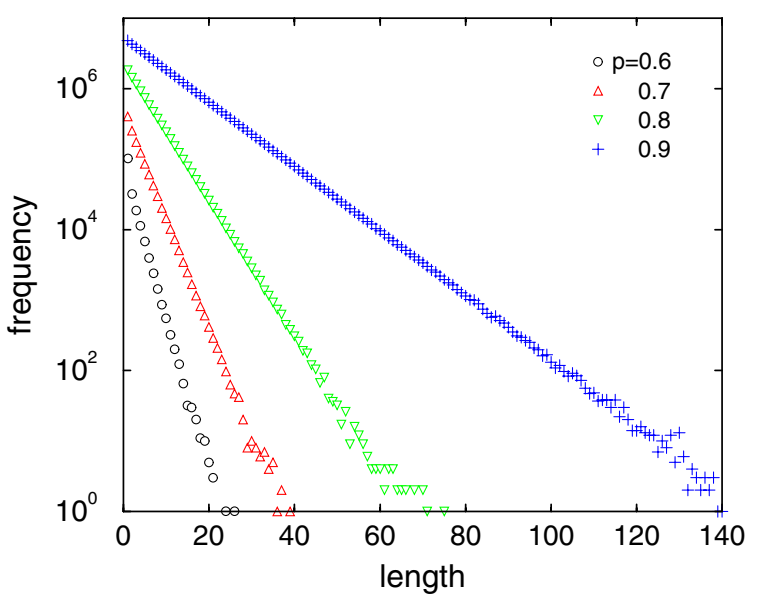

Figure 11. Length distribution of one-cookie domains on a semi-logarithmic scale for 50000 realizations at time $t=37877 \approx 1.5^{26}$ for $p=0.6,0.7,0.8$ and 0.9 .

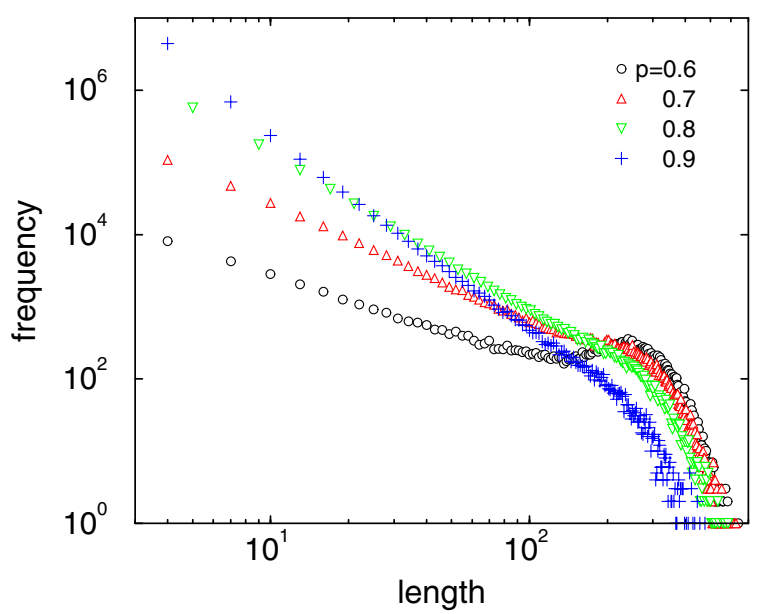

Figure 12. Length distribution of zero-cookie domains on a double logarithmic scale for the same parameters as figure 11 .

neighbouring zero-cookie domains when an intervening one-cookie domain disappears. The zero-cookie domains also have qualitatively different behaviour for $p<p_{c}$ and $p>p_{c}$. In the former case, the distribution has a secondary peak close to the maximum-length domains, while the distribution appears to have a pure power-law form for $p>p_{c}$.

\subsection{Transit time}

Our numerical simulations indicate that the mean displacement $\langle x(t)\rangle$ of the 2-excited walk grows with time as $t^{\nu}$, with $v$ apparently dependent on $p$. For the three cases of $p=0.7,0.8$ and 0.9, least-squares fits to the data in the range $t>20000$ give $v=0.54,0.64$ and 0.85 , respectively. Naively, one might anticipate that the transience of the walk for $p>3 / 4$ would lead to an average displacement that grows linearly with time. Indeed, the argument that gave the threshold bias $p_{c}=3 / 4$ was based on the walk possessing a non-zero bias velocity 


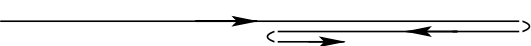

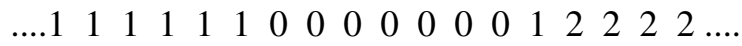

Figure 13. A 2-excited random walk in which the walk has made $k$ consecutive steps to the left after a long run of rightward steps. The corresponding cookie configuration is also shown.

proportional to $p-q$ for $p>p_{c}$. On the other hand, it has been proven that the mean velocity of the 2-excited walk, defined as $\lim _{t \rightarrow \infty}\langle x(t)\rangle / t$ equals zero for all $p<1$ [6].

While we do not know how to determine the time dependence of the displacement, we can address the complementary problem of estimating how long it takes a 2-excited walk to traverse a finite interval. We already know, from the 1-excited walk, that the mean time to traverse any cookie-free interval within an infinite system is divergent. Thus we now consider the time for a 2-excited walk to traverse a finite cookie-free interval. As we shall show, the transit time grows faster than linearly in the interval length when $p$ is close to but strictly less than 1 .

The 2-excited random walk starts at $x=0$ and we compute the time to reach $x=L$, where $L \gg(1-p)^{-k}$. This inequality ensures that a 'defect', namely, a region where the walk takes a small number $k$ of consecutive steps to the left after a long string of rightward steps, will certainly occur in the interval. This defect represents the lowest-order perturbation to the purely ballistic motion for the 2-excited walk when $p \rightarrow 1$. After the defect has been completed and a step to the right has eventually been made, the walk finds itself at the point $x=2$ in a cookie-free region $[0, k]$. We ask: what is the mean time for the walk to reach the right edge of this interval? As illustrated in figure 13, this system is a 1-excited random walk in disguise.

We denote by $T_{k}$ the mean time for the 2-excited walk to reach the point $x=k$ in this geometry. To simplify the calculation, we assume that whenever the left edge of the domain is reached and the last cookie is eaten, the next step of the walk is always to the right. Because this next step could be to the left, our approach represents a lower bound to the transit time that we denote by $T_{k}^{(\mathrm{LB})}$.

As defined in equation (1) and (2), with probability $E_{+}(k, 2)$, the walk reaches $x=k$ without touching $x=0$ and the time for this event is $t_{+}(k, 2)$. On the other hand, with probability $E_{-}(k, 2)$ the walk hits the left edge and then, according to our approximation, hops one step to the right. Since this second argument always equals 2 in all formulae involving $E_{ \pm}$and $t_{ \pm}$, we henceforth drop this argument for simplicity. After these two events, the walk is a distance 2 from the left edge of a cookie-free domain of length $k+1$ and the elapsed time is $t_{-}(k)+1$. Thus $T_{k}^{(\mathrm{LB})}$ obeys the recursion

$$
T_{k}^{(\mathrm{LB})}=E_{+}(k) t_{+}(k)+E_{-}(k)\left[t_{-}(k)+1+T_{k+1}^{(\mathrm{LB})}\right] .
$$

If we allowed the walk to hop to the left (with probability $q$ ) when the leftmost cookie is reached, then equation (40) would be replaced by the exact equation

$T_{k}^{(\mathrm{ex})}=E_{+}(k) t_{+}(k)+E_{-}(k)\left[t_{-}(k)+p\left(1+T_{k+1}^{(\mathrm{ex})}\right)+q p\left(2+T_{k+2}^{(\mathrm{ex})}\right)+q^{2} p\left(3+T_{k+3}^{(\mathrm{ex})}\right)+\cdots\right]$.

The additional terms represent the contribution of paths in which the random walk makes 1 , 2,3 , etc. consecutive steps to the left upon hitting the last cookie before a step to the right is made. This exact equation is not recursive and for simplicity we therefore consider the approximation recursion for the lower bound in what follows. 
By iterating (40), we express $T_{k}^{(\mathrm{LB})}$ compactly as

$T_{k}^{(\mathrm{LB})}=\tau_{k}+\sum_{\ell=1}^{\infty} \tau_{k+\ell} \prod_{j=1}^{\ell} E_{-}(k+j-1)=\tau_{k}+\sum_{\ell=1}^{\infty} \prod_{j=1}^{\ell} E_{-}(k+j-1)$

where $\tau_{k}=E_{-}(k) t_{-}(k)+E_{+}(k) t_{+}(k)=2(k-2)$ is the unconditional exit time, namely, the time for the random walk to exit either side of the interval $[0, k]$ when the starting point is $x=2$. Using this result for $\tau_{k}$, as well as that for $E_{-}(k)$ in equation (41), we obtain after straightforward algebra:

$$
T_{k}^{(\mathrm{LB})}=3(k-2)+2(k-1)(k-2) \sum_{j=k}^{L} \frac{1}{j} .
$$

The upper limit in the sum accounts for case where a cookie-free defect of length $k$ occurs close to the right edge of the domain $[0, L]$ so that terms of order $L$ are needed to account for the growth of the cookie-free defect. Thus we infer that mean time for a 2-excited walk to traverse a finite system of length $L$ scales at least as fast as $L \ln L$. This result is then consistent with $\langle x(t)\rangle$ growing slower than linearly with $t$.

\section{Summary}

The excited random walk is a very rich stochastic process in which the random walk motion is influenced by a transitory bias. Each site of a lattice is initially populated with a finite number of cookies. When a walk reaches a site that contains cookies, the walk eats the cookie and there is a $p / q$ right/left bias at the next step. On the other hand, the walk undergoes unbiased motion if the visited site does not contain cookies. Thus as the cookies are eaten, the bias gradually disappears. If a walk is recurrent, then the interplay between the recurrence and the bias is particularly delicate and the excited random walk will have very different behaviour from pure diffusion or from diffusion with a constant bias. For this reason, the excited random walk appears to be most interesting in one dimension.

In the simplest case of the 1-excited walk on the positive half-line, we employed a physical approach, complementary to that of Dickman et al [10, 11], to show that the first-passage probability for the walk to hit the origin at time $t$ decays as $t^{-1-q}$ for any $q>0$. Thus the 1 -excited walk is always recurrent, except for the trivial case of perfect bias $p=1$. There is especially paradoxical behaviour in the case of $p<1 / 2$, where the cookie-induced bias is directed towards the origin. Here the walk exhibits two seemingly incompatible features: the mean time for the walk to hit the origin is infinite, while the walk strays only a finite distance from the origin during this infinite excursion.

On the infinite line, the 1-excited walk has the same first-passage characteristics as on the semi-infinite line. However, the probability distribution of the walk and the probability distributions for the positions of the last cookie exhibit unusual features. For the probability distribution of the walk, there appears to be a singularity at the origin that does not obey scaling while the rest of the distribution does scale. There is also an asymmetry in the span of the walk that we were able to characterize by a probabilistic argument. The probability distributions for the positions of the leftmost and rightmost cookies have power-law tails that we have been unable, thus far, to explain. These tails seem to be connected with the power-law behaviour for the first-passage probability itself.

The 2-excited walk shows a wide range of strange phenomena. A transition between (conventional) recurrence and transience occurs as $p$ passes through a threshold value $p_{c}=3 / 4$. For $p<p_{c}$, the 2-excited walk appears to qualitatively resemble the 1-excited 
walk. On the other hand, for $p>p_{c}$, the 2-excited walk is transient and this attribute appears responsible for several unexpected features, such as the lack of scaling in the probability distribution of the walk and in the spatial distribution of cookies, as well as the existence of very different distributions of 1-cookie and 0-cookie domains. Most of these features do not yet have clean explanations and the model is ripe for further exploration.

\section{Acknowledgments}

We thank E Ben-Naim and P Krapivsky for useful discussions. TA gratefully acknowledges financial support from the Swiss National Science Foundation under the fellowship 8220067591. SR acknowledges financial support from NSF grants DMR0227670 (at BU) and DOE grant W-7405-ENG-36 (at LANL).

\section{Appendix. Master equation}

In this appendix, we outline a Master equation approach to determine the survival probability of the 1-excited random walk on the infinite half-line. We merely follow the method introduced by Dickman et al $[10,11]$ to describe an unbiased walk between a fixed absorbing boundary at $x=0$ and a moving reflecting boundary that moves to the right with a specified probability each time it is hit by the walk. This perturbed random walk motion is essentially the same mechanism as that of the excited random walk. While the excited walk is ostensibly not Markovian, the process can be made Markovian by considering an extended state space that is defined by the position of the walk $x$ and also the positions of the leftmost and rightmost cookies, $\bar{y}$ and $y$. The exact master equation for the joint probability distribution of the position of the walk and the positions of the last cookies $P(x, \bar{y}, y, t)$ can be obtained in terms of a Markov process.

We consider the simpler problem of the 1-excited random walk with an absorbing boundary at the origin. The system is now described by the joint probability for the cookie to be at $x$ and the leftmost cookie to be at $y, P(x, y, t)$. This distribution obeys the recursion formula

$$
P(x, y, t+1)=\frac{1}{2} P(x-1, y, t)+\frac{1}{2} P(x+1, y, t),
$$

for $x=1,2, \ldots, y-3$. At the origin there is an absorbing boundary condition

$$
P(0, y, t)=0,
$$

while the effect of the cookies is described by slightly modified equations for the probability when the walk is sufficiently close to the position of the last cookie

$$
\begin{aligned}
& P(y-2, y, t+1)=\frac{1}{2} P(y-3, y, t)+\frac{1}{2} P(y-1, y, t)+q P(y-1, y-1, t) \\
& P(y-1, y, t+1)=\frac{1}{2} P(y-2, y, t) \\
& P(y, y, t+1)=\frac{1}{2} P(y-1, y, t)+p P(y-1, y-1, t) .
\end{aligned}
$$

These equations are defined over the wedge domain $x \geqslant 0, x \leqslant y$. We choose the initial conditions that the walk starts at $x=1$ and that the last cookie is also at $x=1$; thus $P(x, y, 0)=\delta_{x, 1} \delta_{y, 1}$. These equations can be iterated then numerically and this is the method that we used for the simulations presented in section II.

From these Master equations, we compute the probability for the excited random walk to reach the origin for the first time at time $t$, from which the survival probability of the walk follows. We merely outline this derivation as it is very similar to that given by Dickman et al $[10,11]$. We first rewrite the Master equations in terms of the generating function 
$\hat{P}(x, y, z)=\sum_{t=0}^{\infty} z^{t} P(x, y, t)$. In this representation, the solution can be written in the product form

$$
\hat{P}(x, y, z)=\hat{A}(x, z) \hat{B}(y, z),
$$

where

$$
\hat{A}(x, z)=\lambda^{x}-\lambda^{-x}, \quad \text { with } \quad \lambda=\frac{1}{z}+\sqrt{\frac{1}{z^{2}}-1,}
$$

and where $\hat{B}$ is defined recursively by

$$
\frac{\hat{B}(y+1, z)}{\hat{B}(y, z)}=\frac{\tanh \Lambda y+2 q \Lambda}{\tanh \Lambda y+\Lambda},
$$

in the $\epsilon \equiv z-1 \rightarrow 0$ limit, with $\Lambda=\ln \lambda$.

The long-time behaviour of the survival probability $\hat{S}(z)=\sum_{x, y} P(x, y, z)$ can now be obtained from the $z \rightarrow 1$ limit $(\Lambda \rightarrow 0)$ of $P(x, y, z)$. For any finite $y$, and thus for $y=y_{0} \sim 2(p-1), \hat{B}\left(y_{0}\right) \sim \hat{B}(1) \sim 1 / \Lambda$ as $\Lambda \rightarrow 0$. On the other hand, for $y>y_{0}$ we approximate the recursion formula (A.6) by

$$
\begin{aligned}
\ln \frac{\hat{B}(y)}{\hat{B}\left(y_{0}\right)} & =\sum_{k=y_{0}}^{y-1} \ln \frac{1+2(p-1) \Lambda / \tanh \Lambda k}{1+\Lambda / \tanh \Lambda k} \\
& \approx(2 p-3) \Lambda \sum_{k=y_{0}}^{y-1} \frac{1}{\tanh \Lambda k} \\
& \approx(3-2 p) \ln \frac{\sinh \Lambda y_{0}}{\sinh \Lambda y}
\end{aligned}
$$

Putting these terms together we obtain

$$
\begin{aligned}
\hat{S}(z) & =\sum_{y=1}^{\infty} \hat{B}(y, z) \sum_{x=0}^{y} \hat{A}(x, z) \\
& \sim \sum_{y=1}^{\infty} \hat{B}(y, z) \frac{4}{\Lambda} \sinh ^{2} \frac{\Lambda y}{2} \\
& \sim \Lambda^{1-2 p} \sum_{y=y_{0}}^{\infty} \frac{\sinh ^{2} \Lambda y / 2}{\sinh ^{3-2 p} \Lambda y} \\
& \sim \Lambda^{-2 p} \int_{\Lambda y_{0}}^{\infty} \frac{\sinh ^{2} u / 2}{\sinh ^{3-2 p} u} \mathrm{~d} u .
\end{aligned}
$$

Since the integral above goes to a finite constant as $\Lambda \rightarrow 0, \hat{S}(z) \sim \Lambda^{-2 p} \sim \epsilon^{-p}$, which corresponds to $S(t) \sim t^{-q}$.

For the 1-excited random walk on the infinite line the phase space is now the threedimensional domain defined by $\bar{y} \leqslant x \leqslant y$. In principle, we can write recursion formulae similar to equations (A.1)-(A.3) for $P(x, \bar{y}, y, t)$, but the solution to these equations no longer seems practical.

\section{References}

[1] Perman M and Werner W 1997 Probab. Theory Relat. Fields 108 357-83

[2] Davis B 1999 Probab. Theory Relat. Fields 113 501-18 
[3] Pemantle R Random processes with reinforcement, Preprint www.math.upenn.edu/ pemantle/papers/ Papers.html.

[4] Benjamini I and Wilson D B 2003 Electr. Commun. Probab. 8 86-92

[5] Angel O, Benjamini I and Virág B 2003 Electr. Commun. Probab. 8 6-16

[6] Zerner M P W 2004 Preprint math.PR/0403060

[7] Madras N and Slade G 1992 The Self-Avoiding Walk (Boston, MA: Birkhauser)

[8] Amit D J, Parisi G and Peliti L 1983 Phys. Rev. B 271635

Tóth B 1994 J. Stat. Phys. 7717

Tóth B 1995 Ann. of Probability 231523

[9] Pemantle R 1992 Probab. Th. Rel. Fields 92117

Pemantle R and Volkov S 1999 Ann. Probab. 271368

[10] Dickman R and ben-Avraham D 2001 Phys. Rev. E 64 020102(R)

[11] Dickman R, Araujo F F Jr. and ben-Avraham D 2003 Braz. J. Phys. 33450

[12] Feller W 1968 An Introduction to Probability Theory and Its Applications (New York: Wiley)

[13] Redner S 2001 A Guide to First-Passage Processes (New York: Cambridge University Press)

[14] Similar arguments were used in Antal T, Hilhorst H J and Zia R K P 2002 J. Phys. A: Math. Gen. 358145

[15] Graham R L, Knuth D E and Patashnik O 1989 Concrete Mathematics: A Foundation for Computer Science (Reading, MA: Addison-Wesley)

[16] Abramowitz M and Stegun I A 1970 Handbook of Mathematical Functions (New York: Dover)

[17] Graham R L, Knuth D E and Patashnik O 1989 Concrete Mathematics: A Foundation for Computer Science (Reading, MA: Addison-Wesley)

[18] Weiss G H 1994 Aspects and Applications of the Random Walk (Amsterdam: North-Holland)

[19] Gradshteyn I S and Ryzhik I M 1965 Tables of Integrals, Series, and Products (New York: Academic) 